\title{
ARTICLE
}

\section{Rapid Scheme of Producing Generalized Fourier Expansion of Matrix Functions and its Application to Physical Problems}

\author{
Toma MATSUMOTO ${ }^{1}$, Yoko YAMANE ${ }^{1}$, Atsunori TANAKA $^{2}$, \\ Shigetoshi SOTA ${ }^{3}$ and Masaki ITOH ${ }^{1, *}$ \\ ${ }^{1}$ Department of Materials Science, Shimane University, Matsue 690-8504, Japan \\ ${ }^{2}$ Center of Nuclear Power-Environmental Study, Shimane Institute of Public Health and Environmental Science, \\ Matsue 690-0122, Japan \\ ${ }^{3}$ Yukawa Institute for Theoretical Physics, Kyoto University, Kyoto 606-8502, Japan
}

\begin{abstract}
Application of orthogonal polynomial expansion to quantum simulations is formulated on a general footing, implementing the regulation technique by Sota and Itoh for treating for the Gibbs oscillation. It is an alternative to the kernel polynomial method using Tchebyshev polynomial, but is simpler to handle and makes it possible to use all the popular orthogonal polynomials, covering finite, semi-infinite and infinite intervals of the eigenvalue spectrum. The accuracy can be made equivalent to direct diagonalization, with the resolution being homogeneous in the whole range of the spectrum. The target quantities can be as diverse as including eigenvectors, as well as all sorts of one-particle properties and correlation functions, involving thermal average and quantum time evolution. It can also be used as a handy tool for solving linear algebraic equations.
\end{abstract}

KEYWORDS: orthogonal polynomial expansion, Gibbs phenomenon, Green function, order-N, eigenvalue spectrum, eigenvector, time evolution, finite temperature correlation function

\section{Introduction}

Numerical evaluation of physical quantities through resolvent matrix has been one of the popular approaches in computational physics. To this category belong the most commonly used continued-fraction technique ${ }^{1)}$ and the kernel polynomial method (KPM) by Voter et al. ${ }^{2-5)}$ Making use of the threeterm recurrence formula of the orthogonal polynomial, a number of advantages may be recognized with the latter method, in terms of the accuracy and the absence of uncertain parameters like the terminator. Being basically a generalized Fourier expansion, the central issue is how one can cope with the Gibbs phenomenon, and the use of Tchebyshev polynomial has been regarded as essential for this purpose. ${ }^{4)}$

Recently Sota and Itoh ${ }^{6)}$ used Legendre polynomial as the basis set, proposing an alternative way to remove Gibbs oscillation. It is called the regulated polynomial expansion (RPE) and has a very different principle. While the KPM modifies the expansion coefficients to suppress the fluctuations, the RPE modifies the basis polynomials by the procedure called regulation, in such a way that the oscillating contributions from different expansion orders are made to cancel entirely. The degree of each polynomial is unaltered, but the amplitude is modified and the zeros are shifted relative to the original locations. Since the regulated polynomials are produced recursively, the algorithm of the RPE is essentially the same as the naive polynomial expansion, requiring no additional routines to remove Gibbs phenomenon.

\footnotetext{
*Corresponding author, E-mail: itomasa@riko.shimane-u.ac.jp
}

Due to the simplicity and even higher controllability of resolution, the RPE is expected to extend vastly the range of physical quantities to be handled by the polynomial expansion. In the original paper, it has been applied to the calculation of the eigenvectors, with the accuracy being equivalent to the direct diagonalization. Applications to the electron transport and Anderson localization were then attempted. ${ }^{7,8)}$ Further the scheme was transferred to the imaginary-time domain, being applied to the finite-temperature calculations of correlation functions of strongly correlated electrons and frustrated spin system, combined with the technique of the density matrix renormalization group (DMRG). ${ }^{11-13)}$

In view of the above ongoing developments and further potential applicability, we attempt in the present paper to formulate the method on a general ground, without specifying the basis polynomial. This generalization enables us to make use of all the polynomials, defined not only in a finite but also in semi-infinite and infinite intervals of the eigenvalue spectrum. Reviewing our earlier results, we examine the working order of the method more closely, clarifying its mathematical structure and fundamental properties of the kernel function. A particular emphasis is laid on resolvent, but we also discuss various extensions from a unified viewpoint. Particularly the time-evolution operator is carefully examined with some numerical studies.

The paper is organized as follows. In Section II we briefly summarize the polynomial expansion of resolvent expansion. The mathematical structure is studied carefully, as well as its 
recursive structure and relationship to the moment expansion. In Section III we formulate the regulation technique, and show some numerical examples to see how effectively it removes the Gibbs phenomenon. Various applications are discussed in Section IV, including the calculations of eigenvectors and quantum time evolution. The final section is devoted to a summary. Some mathematical details are described in Appendix A. In Appendix B, we tabulate all the necessary inputs for invoking the RPE. Although the paper is rather intricate mathematically, a quick reference to this table will suffice for using the method.

\section{General Framework of Polynomial Expansion and its Application to Resolvent}

In this section we formulate the naive polynomial expansion of resolvent, without caring for the Gibbs phenomenon. The latter problem will be discussed in the next section. We shall give in Appendix A the explicit expressions for the expansion coefficients, for all the popular polynomials, with detailed proof for each. Here we only outline the mathematical argument and present a practical way to calculate them.

We only demand the three basic properties for the polynomial set:

$$
\begin{gathered}
\int_{a}^{b} d x w(x) \varphi_{n}(x) \varphi_{n^{\prime}}(x)=w_{n} \delta_{n, n^{\prime}}, \\
\delta\left(x-x^{\prime}\right)=w(x) \sum_{n} w_{n}^{-1} \varphi_{n}(x) \varphi_{n}\left(x^{\prime}\right)
\end{gathered}
$$

and

$$
\varphi_{n+1}(x)=\left(A_{n} x+B_{n}\right) \varphi_{n}(x)+C_{n} \varphi_{n-1}(x) .
$$

Here $\varphi_{n}(x)$ represents a polynomial of degree $n$ belonging to the basis set, $[a, b]$ is its definition interval, $w_{n}$ the normalization constant and $w(x)$ the weight function. To this family belong Legendre, Tchebyshev, Jacobi, Laguerre and Hermite polynomials, but we can make use of many others, as long as the conditions (1)-(3) are satisfied.

Now suppose that we wish to handle the operation of a function of a quantum operator $\hat{\Omega}, f(\hat{\Omega})$, on a vector $\mid \xi>$. The matrix size of $\hat{\Omega}$ may be quite large, involving many degrees of freedom. Typical examples are the functions of the Hamiltonian $\hat{H}$, e.g. the kernel operator $\delta(\epsilon-\hat{H})$, resolvent $[z-\hat{H}]^{-1}$, the time evolution operator $e^{-i \hat{H} t}$ or the density matrix $e^{-\beta \hat{H}}$. We expand $f(\hat{\Omega})$ as

$$
f(\hat{\Omega})=\sum_{n=0}^{\infty} w_{n}^{-1} f_{n} \varphi_{n}(\hat{\Omega}),
$$

where

$$
f_{n}=\int_{a}^{b} d x w(x) \varphi_{n}(x) f(x) .
$$

Then handling the operation $f(\hat{\Omega}) \mid \xi>$ is easy, because each term $\varphi_{n}(\hat{\Omega}) \mid \xi>$ is evaluated recursively using (3),

$$
\begin{aligned}
& \varphi_{n+1}(\hat{\Omega}) \mid \xi> \\
& =\left(A_{n} \hat{\Omega}+B_{n}\right) \varphi_{n}(\hat{\Omega})\left|\xi>+C_{n} \varphi_{n-1}(\hat{\Omega})\right| \xi>.
\end{aligned}
$$

This leads to an ideal order-N scheme, because only $N$ matrixvector (not matrix-matrix) multiplications need to be executed for the truncation order $N$. In practice, we need to know the expansion coefficients $f_{n}$ for each function $f$. We will discuss the case of resolvent in this section. Other cases are relatively easy.

\section{Resolvent and Adjoint Functions}

In many branches in physics, Green function method is one of the most useful techniques. It uses resolvent operator

$$
\hat{G}(z)=(z-\hat{H})^{-1},
$$

where $\hat{H}$ is the Hamiltonian and $z$ the complex frequency parameter. This corresponds to $f(x)=(z-x)^{-1}$ and $\hat{\Omega}=\hat{H}$ in Eq. (4), and our concern is how one can evaluate its matrix elements effectively. Equations (4) and (5) then read

$$
\hat{G}(z)=\sum_{n=0}^{\infty} w_{n}^{-1} \tilde{\varphi}_{n}(z) \varphi_{n}(\hat{H})
$$

and

$$
\tilde{\varphi}_{n}(z)=\int_{a}^{b} d x \frac{w(x) \varphi_{n}(x)}{z-x}
$$

respectively. Here we have used the symbol $\tilde{\varphi}_{n}(z)$ instead of $f_{n}$, in order to show explicitly the dependence on $z$ and the relevance to $\varphi_{n}(x)$. We call $\tilde{\varphi}_{n}(z)$ the adjoint function $(A F)$ to $\varphi_{n}(x)$. In practice, we need their analytical continuations $\tilde{\varphi}_{n}(\epsilon \pm i \delta)$ onto the real axis. We will discuss it in some detail in the following two subsections. It is obvious that their imaginary parts are given by

$$
\operatorname{Im} \tilde{\varphi}_{n}(\epsilon \pm i \delta)=\mp \pi w(\epsilon) \varphi_{n}(\epsilon) .
$$

The corresponding part of resolvent,

$$
\delta(\epsilon-\hat{H})=w(\epsilon) \sum_{n=0}^{\infty} w_{n}^{-1} \varphi_{n}(\epsilon) \varphi_{n}(\hat{H}),
$$

is called the kernel operator.

The expansion (8) has some relevance to the moment expansion. The AF of the $n$-th degree is expanded around the infinity as

$$
\tilde{\varphi}_{n}(z)=\sum_{m=n}^{\infty} \frac{K_{m}^{(n)}}{z^{m+1}}
$$

with the coefficients $K_{m}^{(n)}$ defined by

$$
K_{m}^{(n)}=\int_{a}^{b} d x x^{m} w(x) \varphi_{n}(x) .
$$

Note that the summation over $m$ in Eq. (12) is restricted to $m \geq n$, since $K_{m}^{(n)}=0$ for $m<n$ due to the orthogonality of the polynomials. This leads to an important asymptotic condition at the infinity,

$$
\lim _{|z| \rightarrow \infty} z^{n+1} \tilde{\varphi}_{n}(z)=K_{n}^{(n)} .
$$


When the expansion (8) is truncated at some finite order $N$, Eqs. (8) and (12) may be written as

$$
\hat{G}_{N}(z) \equiv \sum_{n=0}^{N} w_{n}^{-1} \tilde{\varphi}_{n}(z) \varphi_{n}(\hat{H})=\sum_{m=0}^{\infty} \frac{\hat{\mu}_{m}}{z^{m+1}}
$$

with

$$
\hat{\mu}_{m} \equiv \sum_{n=0}^{N} K_{m}^{(n)} \varphi_{n}(\hat{H}) .
$$

Here the subscript of $\hat{G}_{N}$ is to indicate the truncation order. The trace of the operator $\hat{\mu}_{m}$ gives an approximate expression to the $m$-th order moment of the frequency spectrum. It is actually exact for $m \leq N$, because $\hat{\mu}_{m}$ is then identical to $\hat{H}^{m}$. So the moments of the DOS are conserved strictly up to the order of the truncation. For higher-orders $(m>N)$, Eq. (16) still gives an optimal approximation to $\hat{H}^{m}$, in the sense that it minimizes the squared norm $\left\|\hat{\mu}_{m}-\hat{H}^{m}\right\|^{2}$ with a limited number of polynomials, which is the major difference from the moment expansion.

\section{Determination of Adjoint Functions}

The determination of the AF through the integration (9) is generally not elementary. Particularly one must be careful about its proper analytical continuation to obtain $\tilde{\varphi}_{n}(\epsilon \pm i \delta)$. However it can be carried through via a different root. The argument is fairly involved, and we give here only a brief account, taking Jacobi polynomial as a representative case. A thorough discussion is given in the Appendix A.

First, we note that Eq. (9) may be regarded as a special case of Hilbert transformation

$$
\psi(z)=\int_{a}^{b} d x(z-x)^{-1} v(x) .
$$

This transformation allows one to express differentiations on $\psi(z)$ by those on $v(x)$, by partial integrations. In particular, when $\psi(z)$ satisfies a canonical second-order differential equation $\mathcal{L}_{z} \psi(z)=0, v(x)$ is shown to satisfy the same equation $\mathcal{L}_{x} v(x)=0$, if the marginal contributions resulting from the partial integrations vanish. ${ }^{14)}$ This is indeed the case for all the popular polynomials (see Appendix A), so we can readily derive the differential equation for $\tilde{\varphi}_{n}(z)$ from that for $\varphi_{n}(z)$. We call it the adjoint differential equation $(A D E)$. In the case of Jacobi polynomial $P_{n}^{\alpha \beta}(x)$, the ADE becomes

$$
\begin{aligned}
& \left(z^{2}-1\right) \frac{d^{2}}{d z^{2}} \tilde{P}_{n}^{\alpha \beta}(z) \\
& -[(\alpha+\beta-2) z+(\alpha-\beta)] \frac{d}{d z} \tilde{P}_{n}^{\alpha \beta}(z) \\
& \quad-(n+1)(n+\alpha+\beta) \tilde{P}_{n}^{\alpha \beta}(z)=0 .
\end{aligned}
$$

Here we have denoted by $\tilde{P}_{n}^{\alpha \beta}(z)$ the AF associated with $P_{n}^{\alpha \beta}(x)$. The same convention will also be adopted for other polynomials throughout this paper. Other ADE's are readily written down for the respective polynomials. Generally, they are given in Papperitz canonical forms, with the three regular singular points at $z=a, b$ and $z=\infty$, but it can happen that two of these singularities are merged into a confluent type.

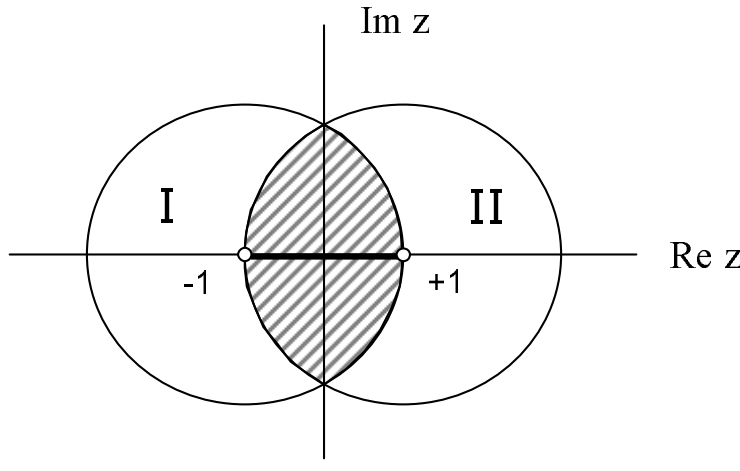

Fig. 1 Convergence circles of the two functions (50) and (52) leading to the two expressions in Eq. (19). They are identical in the common area (hatched). The bold line connecting $z= \pm 1$ is the branch cut.

The physical solution to $\tilde{\varphi}_{n}(z)$ is generally constructed by taking a linear combination of the two independent solutions of the ADE, one of which may be taken to be $w(z) \varphi_{n}(z)$. In doing so, the asymptotic condition (14) is to be consulted to determine the combination uniquely. It is then analytically continued onto the real axis to obtain $\tilde{\varphi}_{n}(\epsilon \pm i \delta)$. The continuation yields two branching points at $z=a$ and $z=b$, as well as the branch cut connecting them. The case of Jacobi polynomial is a little intricate, because two different functions can result from this procedure, i.e., Eqs. (50) and (52). They are identical in the common area of the two convergence circles shown in Fig. 1, and two alternative expressions are obtained,

$$
\begin{aligned}
& \tilde{P}_{n}^{\alpha \beta}(\epsilon \pm i \delta) \\
& =[\pi \cot (\pi \beta) \mp i \pi] \cdot(1-\epsilon)^{\alpha}(1+\epsilon)^{\beta} P_{n}^{\alpha \beta}(\epsilon) \\
& +2^{\alpha+\beta}(-1)^{n+1} \frac{\Gamma(\beta) \Gamma(\alpha+n+1)}{\Gamma(\alpha+\beta+n+1)} \\
& \quad \times F\left(n+1,-\alpha-\beta-n|1-\beta| \frac{1+\epsilon}{2}\right) \\
& =[-\pi \cot (\pi \alpha) \mp i \pi] \cdot(1-\epsilon)^{\alpha}(1+\epsilon)^{\beta} P_{n}^{\alpha \beta}(\epsilon) \\
& +2^{\alpha+\beta} \frac{\Gamma(\alpha) \Gamma(\beta+n+1)}{\Gamma(\alpha+\beta+n+1)} \\
& \quad \times F\left(n+1,-\alpha-\beta-n|1-\alpha| \frac{1-\epsilon}{2}\right),
\end{aligned}
$$

where $F(a, b|c| z)$ denotes the hypergeometric function.

There is another complication in Eqs. (19): in either of the two expressions, the two separate terms involve divergences respectively, when either of the two indices $(\alpha, \beta)$ is an integer. It is apparent for the first terms due to the co-tangents. A similar divergence also occurs in the case of Laguerre, but these problems are spurious and circumvented as shown in the next subsection.

\section{Recursive Structure of Adjoint Functions}

The preceding discussion, together with the complement in Appendix A, clarifies the mathematical structure in the com- 
plex frequency plane. However, evaluating $\tilde{\varphi}_{n}(\epsilon \pm i \delta)$ through the expressions like Eqs. (19) to the higher degrees is not too convenient. Instead, we may make use of the recurrence formulae

$$
\begin{gathered}
\tilde{\varphi}_{n+1}(z)=\left(A_{n} z+B_{n}\right) \tilde{\varphi}_{n}(z)+C_{n} \tilde{\varphi}_{n-1}(z), \\
\tilde{\varphi}_{1}(z)=\tilde{\varphi}_{0}(z) \varphi_{1}(z)-A_{0} w_{0}
\end{gathered}
$$

for evaluating those functions. The above formulae, the first of which takes the same form as Eq. (3), are readily proven by using Eqs. (3) and (9) and noting that the integral $\int_{a}^{b} w(x) \varphi_{n}(x) d x$ vanishes when $n \geq 1$. We thus need only the initial function $\tilde{\varphi}_{0}(\epsilon \pm i \delta)$.

At first sight, the forms of $\tilde{\varphi}_{n}(z)$ for higher degrees anticipated from Eq. (20) may appear at variance with Eq. (12). But the asymptotic form is assured by the following identity

$$
K_{m}^{(n+1)}=A_{n} K_{m+1}^{(n)}+B_{n} K_{m}^{(n)}+C_{n} K_{m}^{(n-1)},
$$

which is proven by multiplying Eq. (12) by $w(x) x^{m}$ and then integrating over $a<x<b$. Note, also, that the branching points of $\tilde{\varphi}_{0}(z)$ are inherited successively to the higher degrees, so that all the functions $\tilde{\varphi}_{n}(z)$ share the same cut.

The initial functions are tabulated in Appendix B, except for the cases of Jacobi and Laguerre polynomials with integral indices. Integral indices are obviously common in comparison to non-integral ones, but cause apparent divergences, as mentioned in the preceding subsection. As for the initial term, however, divergence-free expressions are obtainable directly from (9),

$$
\begin{aligned}
\tilde{P}_{0}^{\alpha \beta}(\epsilon \pm i \delta) & =w(\epsilon) \times\left(\ln \left|\frac{1+\epsilon}{1-\epsilon}\right| \mp i \pi\right) \\
& +\sum_{\mu=1}^{\alpha} \frac{2^{\alpha+\beta}}{\alpha-\mu+1}\left(\frac{1-\epsilon}{2}\right)^{\mu-1}\left(\frac{1+\epsilon}{2}\right)^{\beta} \\
& -\sum_{\nu=1}^{\beta} \frac{2^{\alpha+\beta} \alpha !(\beta-\nu) !}{(\alpha+\beta+1-\nu) !}\left(\frac{1+\epsilon}{2}\right)^{\nu-1}
\end{aligned}
$$

and

$$
\begin{aligned}
\tilde{L}_{0}^{\alpha}(\epsilon \pm i \delta) & =w(\epsilon) \times(-E i(\epsilon) \mp i \pi) \\
& -\sum_{\mu=1}^{\alpha}(\alpha-\mu) ! \epsilon^{\mu-1}
\end{aligned}
$$

where $w(\epsilon)$ represents $(1-\epsilon)^{\alpha}(1+\epsilon)^{\beta}$ for Jacobi and $\epsilon^{\alpha} e^{-\epsilon}$ for Laguerre. Equation (23) coincides with the result for Legendre when $\alpha=\beta=0$ (Appendix B), for which only the first term is relevant.

\section{Gibbs Oscillation and Regulation}

\section{Regulated Polynomial}

The argument in the preceding section completes the formal polynomial expansion of resolvent. The problem of the Gibbs phenomenon is yet to be overcome. We show in Fig. 2 the frequency spectrum of a lattice vibration, calculated by

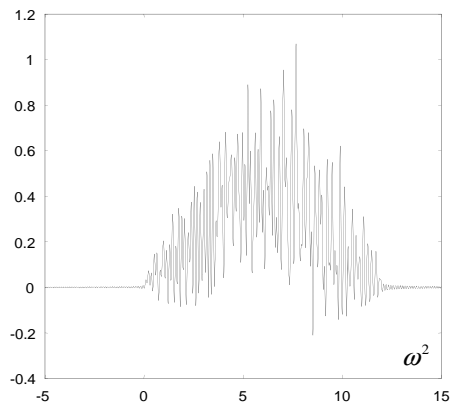

Fig. 2 The eigenvalue spectrum of a SC lattice vibration of $19^{3}$ atoms, without treatment for the Gibbs oscillation. Legendre polynomial is used with the truncation $N=10^{3}$

using the expansion (11) with Legendre polynomial. The system we studied is a simple-cubic lattice of $19^{3}$ atoms with the nearest neighbour force constant. The periodic boundary condition has been adopted for constructing the dynamical matrix. The size of the matrix is typical of ordinary numerical studies, and the bulk spectrum of the model is calculable analytically. As is seen from the figure, most of the important physical information is lost by the violent fluctuations. The problem is actually in two ways; firstly due to the Gibbs phenomenon, and secondly due to the discrete nature of the spectrum. It is therefore important, for a modest size of the matrix, to distinguish between the two different effects numerically.

Sota and Itoh ${ }^{6)}$ showed that the former effect is entirely eliminated by using the regulated polynomial

$$
\left\langle\varphi_{n}(x)\right\rangle_{\sigma} \equiv \frac{1}{\sqrt{2 \pi \sigma^{2}}} \int_{a}^{b} d x^{\prime} e^{-\left(x^{\prime}-x\right)^{2} / 2 \sigma^{2}} \varphi_{n}\left(x^{\prime}\right)
$$

as the new basis set, instead of the original polynomial set $\varphi_{n}(x)$. The resultant expressions for resolvent and kernel operators are

$$
\left\langle\hat{G}_{N}(z)\right\rangle_{\sigma}=\sum_{n=0}^{N} w_{n}^{-1} \tilde{\varphi}_{n}(z)\left\langle\varphi_{n}(\hat{H})\right\rangle_{\sigma}
$$

$$
\left\langle\delta_{N}(\epsilon-\hat{H})\right\rangle_{\sigma}=w(\epsilon) \sum_{n=0}^{N} w_{n}^{-1} \varphi_{n}(\epsilon)\left\langle\varphi_{n}(\hat{H})\right\rangle_{\sigma} .
$$

This is the regulated polynomial expansion (RPE). Its effectiveness is illustrated in the next subsection. For its practical use, the above authors noted that $\left\langle\varphi_{n}(x)\right\rangle_{\sigma}$ may be regarded as a polynomial of $n$-th degree for sufficiently small $\sigma$, since the integration in Eq. (25) can be practically extended to $-\infty<x^{\prime}<\infty$. Further they found that these "pseudo polynomials" may be evaluated by a set of recursive relations

$$
\begin{aligned}
\left\langle\varphi_{n+1}(x)\right\rangle_{\sigma}= & \left(A_{n} x+B_{n}\right)\left\langle\varphi_{n}(x)\right\rangle_{\sigma}+C_{n}\left\langle\varphi_{n-1}(x)\right\rangle_{\sigma} \\
& +\sigma^{2} A_{n}\left\langle\varphi_{n}^{\prime}(x)\right\rangle_{\sigma}
\end{aligned}
$$

and

$$
\begin{aligned}
& \left\langle\varphi_{n+1}^{\prime}(x)\right\rangle_{\sigma} \\
& \quad=A_{n}^{\prime}\left\langle\varphi_{n}(x)\right\rangle_{\sigma}+B_{n}^{\prime}\left\langle\varphi_{n}^{\prime}(x)\right\rangle_{\sigma}+C_{n}^{\prime}\left\langle\varphi_{n-1}^{\prime}(x)\right\rangle_{\sigma} .
\end{aligned}
$$


In the original paper the above relations were derived only for Legendre polynomial, but the same kinds are available for all the popular polynomials. The first relation is readily derived from Eq. (6) and Eq. (25). It involves a derivative term coming from $x \varphi_{n}(x)$ in Eq. (3). Note that the differentiation and the regulation may be performed in the reversed order, i.e., $\left\langle\varphi_{n}^{\prime}(x)\right\rangle_{\sigma}=d / d x\left\langle\varphi_{n}(x)\right\rangle_{\sigma}$. The second relation is a direct consequence of the same relation among the unregulated polynomials. The coefficients $A_{n}^{\prime}, B_{n}^{\prime}$ and $C_{n}^{\prime}$ are tabulated in Appendix B for all the popular polynomials, since the authors did not find all of them in the literature.

The above closure relations may be used with a matrix variable in substitution for Eq. (6). This algorithm requires only a minimum modification of the naive expansion. Since only a single matrix-vector multiplication is involved in Eqs. (28) and (29), there is no appreciable change in the computation time. We emphasize that, unlike the conventional methodology for suppressing Gibbs oscillation, the RPE does not merely damp the higher-orders in the expansion. Instead it rather modifies the basis set, in such a way that the oscillating contributions from different orders are made to cancel. For example, it also involves re-location of the zeros of the polynomial.

\section{Kernel Functions and Eigenvalue Spectrum}

The effect of regulation is best illustrated by examining the kernel function, i.e. the eigenvalue of the kernel operator. In Fig. 3, we make comparison between the regulated and unregulated kernel functions, calculated by using Legendre polynomial. The former is given by

$$
\left\langle\delta_{N}\left(\epsilon-\epsilon_{\alpha}\right)\right\rangle_{\sigma}=w(\epsilon) \sum_{n=0}^{N} w_{n}^{-1} \varphi_{n}(\epsilon)\left\langle\varphi_{n}\left(\epsilon_{\alpha}\right)\right\rangle_{\sigma},
$$

where $\epsilon_{\alpha}$ represents an eigenvalue of the Hamiltonian. It may be regarded as a Fourier expansion of the normal distribution function used in Eq. (25), so the RPE kernel is designed to be close to it:

$$
\left\langle\delta_{N}\left(\epsilon-\epsilon_{\alpha}\right)\right\rangle_{\sigma} \simeq \frac{1}{\sqrt{2 \pi \sigma^{2}}} e^{-\left(\epsilon-\epsilon_{\alpha}\right)^{2} / 2 \sigma^{2}} .
$$

As we show in the next subsection, the accuracy of the above approximation is extraordinary for an optimal value of $\sigma$, which can be made arbitrarily small by choosing a larger $N$. The best $\sigma$ is practically independent of the eigenvalue $\epsilon_{\alpha}$.
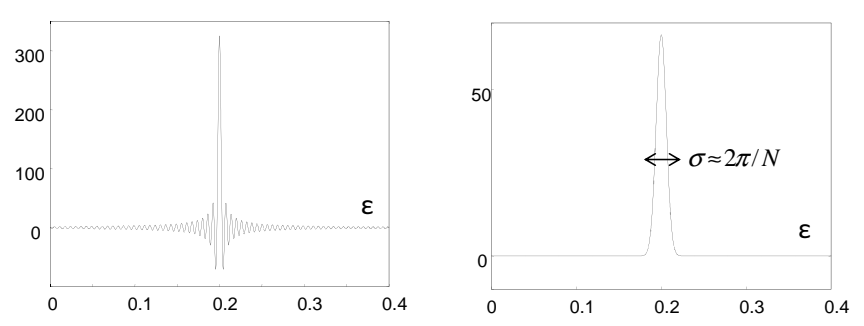

Fig. 3 Comparison between the two kernel functions with Legendre polynomial. Left: without regulation, right: with regulation. Here $\epsilon_{\alpha}=0.2$ and the value of $\sigma$ is taken to be $2 \pi / N$ on the right.
The similarity to the normal distribution is also reported in the case of Jackson kernel in the KPM, ${ }^{4)}$ but there is a fundamental difference between the two cases. First, the latter function is constructed by different principles, so deviates considerably from normal distribution. Also, the variance of Jackson kernel depends explicitly on the eigenvalue, causing an inhomogeneous resolution. ${ }^{4,5)}$ These points will be discussed quantitatively in Section III.3.

Due to the above properties of the RPE kernel, the RPE spectrum

$$
D(\epsilon)=\sum_{\alpha}\left\langle\delta_{N}\left(\epsilon-\epsilon_{\alpha}\right)\right\rangle_{\sigma}
$$

is positive and strictly normalized, with a homogeneous resolution in the whole range of the spectrum. Figure 4 shows its applications to the same phonon spectrum studied in Fig.2, ${ }^{6}$ ) with low and high truncation orders. In each case, the parameter $\sigma$ is set equal to its optimal value $2 \pi / N$ (this value will be confirmed in Section III.3). In either case, the Gibbs oscillation is completely eliminated. For the present matrix size, a relatively low-truncation calculation shown on the left ( $N=200)$ provides a best simulation of the bulk, resembling the analytic solution. The high-truncation calculation on the right, on the other hand, shows the precise spectrum of the finite matrix. The spikes are by no means due to the Gibbs oscillation, but solely to the discreteness of the spectrum. The discrete character starts to show up around $N \simeq 2 \times 10^{5}$; here the resolution has been increased by far. By comparing to the direct diagonalization, the location and the height of each peak have been confirmed to give the correct eigenvalue and degeneracy, to the accuracy of the six digits.

The same calculation has also been attempted with the two Jacobi polynomials, with $\alpha=\beta=-1 / 2$ and $+1 / 2$. The former case is equivalent to using Tchebyshev. The results are indistinguishable from the case of Legendre $(\alpha=\beta=0)$, although a very fine Gibbs oscillation was found to persist in the case of Tchebyshev.
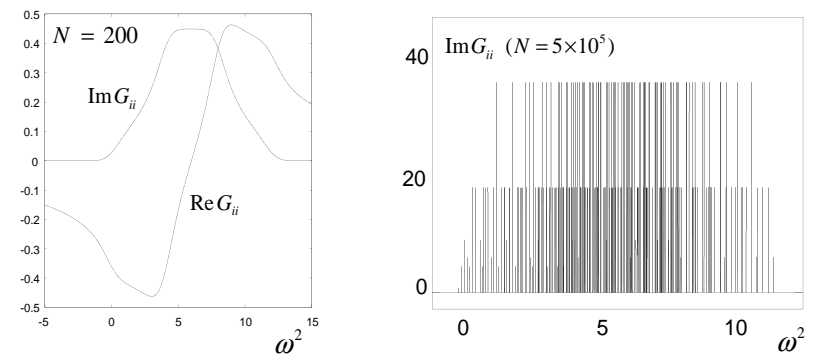

Fig. 4 Re-calculation of the same spectrum as shown in Fig. 2, $\left.{ }^{6}\right)$ using regulated Legendre polynomial. Left: low truncation order $(N=200)$, right: high truncation $\left(N=5 \times 10^{5}\right)$. The resolution is set to be $\sigma=2 \pi / N$ in the both cases.

\section{Optimal Resolution and Kernel Operator}

The optimal value of $\sigma$ can be searched for by minimizing 
the squared norm

$$
\begin{aligned}
I_{N}(\sigma) \equiv \int_{a}^{b} d \epsilon & w(\epsilon) \mid\left\langle\delta_{N}\left(\epsilon-\epsilon_{\alpha}\right)\right\rangle_{\sigma} \\
& -\left.\frac{1}{\sqrt{2 \pi \sigma^{2}}} e^{-\left(\epsilon-\epsilon_{\alpha}\right)^{2} / 2 \sigma^{2}}\right|^{2}
\end{aligned}
$$

with varying $\sigma$ for a given $N$. This integration represents the deviation of the kernel function from the normal distribution. It is plotted in Fig. 5, on the left. As is seen from the figure, the norm decreases rapidly and monotonically as we increase $\sigma$. It goes down to the smallest values that are barely dealt with by double precision. In searching for the best $\sigma$, we have therefore set a criterion that $I_{N}(\sigma) \leq \delta$, where $\delta$ is a small positive number, and chose the smallest $\sigma$ to satisfy this criterion. On the right in the same figure we show the results with $\delta=5 \times 10^{-16}$, a value just starting to cause numerical underflow. We have repeated the above calculation for all the polynomials listed in Appendix B. In every case, $\sigma(N)$ is fitted very precisely by $\sigma(N)=C / N^{\alpha}$. Here the index $\alpha$ is strictly unity for the polynomials defined in the interval $[-1,+1]$, and $1 / 2$ for Laguerre and Hermite. The value of $C$ depends appreciably on $\delta$; there is a tolerable range of $C$ for each $\delta$ and for each polynomial. In the case of Legendre, $\delta=10^{-8}$ corresponds to $4 \leq C \leq 8$. So $C=4$ may be used to attain the highest resolution within this accuracy. Going beyond this range causes eventual blow-up of the norm. It seems that the best value for an oscillationless kernel is obtained by $C=2 \pi$, for all $\delta$.

We have not specified the value of $\epsilon_{\alpha}$ in Fig. 5. Actually we found that $I_{N}(\sigma)$ is practically independent of $\epsilon_{\alpha}$ when $N$ is relatively large; we do not see the difference in the scale oused in Fig. 5. The variation of $I_{N}(\sigma)$ with $\epsilon_{\alpha}$ is comparable to $\delta$, unless the values of $\epsilon_{\alpha}$ are too close to the limits of the definition interval. With such restrictions on $N$ and $\epsilon_{\alpha}$ in mind, the fact implies a completely homogeneous resolution of the RPE spectrum. Also we may express Eq. (31) in the operator form,

$$
\left\langle\delta_{N}(\epsilon-\hat{H})\right\rangle_{\sigma} \simeq \frac{1}{\sqrt{2 \pi \sigma^{2}}} e^{-(\epsilon-\hat{H})^{2} / 2 \sigma^{2}} .
$$

This "analytic" expression is useful in using the RPE in the time or the finite temperature domain (Section IV.5). The accuracy of Eqs. (31) or (34) is assured even for a relatively
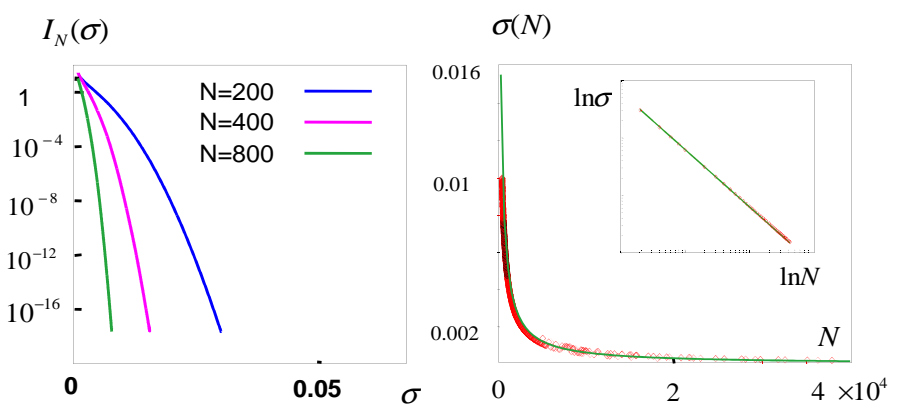

Fig. 5 Left: squared norm (33) for the regulated Legendre kernel, as functions of $\sigma$. Right: smallest $\sigma$ to satisfy $I_{N}(\sigma) \leq 5 \times 10^{-16}$. The calculated values are very well fitted by $\sigma=2 \pi / N$, as shown by the inset on the right.

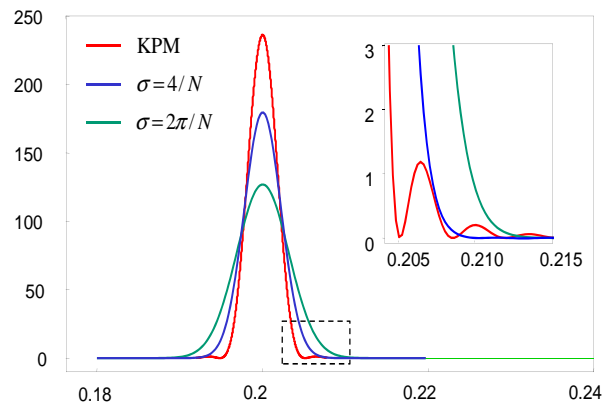

Fig. 6 Comparison between KPM Jackson and Legendre RPE kernels for $N=2000$, with optimal and non-optimal values of $\sigma$ for the latter case. The small oscillations in the RPE kernel pointed out by Schleede et al. ${ }^{9 \text { ) }}$ are not visible in the magnified scale.

small $N$. In this connection we note that the variance of the RPE kernel is fixed strictly to $\sigma$ and the first moment to zero, irrespective of $\epsilon_{\alpha}$ and $N$.

We also note that the above characteristic of the RPE kernel is considerably different than that of the KPM. Being practically identical to the normal distribution, the RPE kernel is evidently oscillationless, as opposed to the criticism raised recently by Schleede et al. ${ }^{9)}$ The resolution is uniform and depends only on $N$. In the KPM, the Jackson kernel becomes asymmetric and varies its width, as the eigenvalue goes away from the center of the spectrum. The squared norm corresponding to Eq. (33) is of the order of unity and increases with increasing $N$. In fact the Jackson kernel carries many satellite peaks representing the uneliminated Gibbs oscillation, as shown in Fig. 6. Those satellite peaks grow with increasing $N$. The above authors claim that residual oscillations exist in the Legendre RPE kernel for $\sigma=4 / N$, but such oscillations are by many orders of magnitude smaller than those satellite peaks, if an optimal $\sigma$ is used; more than two orders smaller even for $\sigma=4 / N$, which is not optimal.

\section{Other Applications}

In Sections II and III we have focused on resolvent and eigenvalue spectrum. In this section we briefly summarize other immediate extensions of the methodology.

\section{One-Particle Properties}

The RPE can be applied to various spectrum-related properties directly. For example, the integrated density of states is calculated as follows:

$$
N(\epsilon)=\sum_{n=0}^{\infty} w_{n}^{-1} \xi_{n}(\epsilon) \operatorname{Tr}\left\langle\varphi_{n}(\hat{H})\right\rangle_{\sigma},
$$

where $\xi_{n}(\epsilon)$ are given by

$$
\xi_{n}(\epsilon) \equiv \int_{a}^{\epsilon} d x w(x) \varphi_{n}(x)
$$


With Rodrigue's formula, the integrand of Eq. (36) becomes a complete differential when $n>0$, which yields

$$
\xi_{n}(\epsilon)= \begin{cases}-\frac{1}{2 n} w(\epsilon) P_{n-1}^{\alpha+1, \beta+1}(\epsilon) & (\text { Jacobi) } \\ -w(\epsilon) H_{n-1}(\epsilon) & \text { (Hermite) } \\ \frac{1}{n} w(\epsilon) L_{n-1}^{\alpha+1}(\epsilon) & \text { (Laguerre) } .\end{cases}
$$

In the above expressions, the weight functions $w(\epsilon)$ are those associated with the indices given on the right hand sides of the equations. The expression (35) avoids the numerical integrations and evaluates the integrated density of states directly. The coefficients $\xi_{n}(\epsilon)$ are also obtainable recursively.

Many of the integrations involving the frequency spectrum (or single resolvent) are treated in similar manners. For electron problems, for instance, the total energy and the higher order moments of the density of states are given in similar forms to Eqs. (35)-(37). A somewhat different (and handy) algorithm was proposed by Sota and Itoh for evaluating them with Legendre polynomial. ${ }^{6}$

\section{Correlation Functions}

A correlation function is one of the most suitable targets of the polynomial expansion technique, and the KPM has often been utilized for this purpose. ${ }^{10)}$ Recently, Sota and Tohyama ${ }^{11,12)}$ and Sugimoto et al. $^{13)}$ have applied the RPE to a finite-temperature calculation, developing a combined scheme with the density matrix renormalization group (DMRG). In these calculations, they deal with the correlation function of the form

$$
\chi_{A B}(\omega) \propto \int d \epsilon\langle\delta(\epsilon-\hat{H}) \hat{A} \delta(\epsilon+\omega-\hat{H}) \hat{B}\rangle,
$$

where $\langle\cdots\rangle$ denotes the finite-temperature statistical average. For calculating a linear-response function like Eq. (38), the RPE requires only the repetition of the same algorithm, for operating different operators successively. The computation time is proportional only to the number of the functional operators involved. In principle, there is no limitation in the number of such operators, so a complex property such as the Hallconductivity can be a target quantity. ${ }^{15)}$ For taking a thermal average, the density matrix $e^{-\beta \hat{H}}$ and/or the (imaginary) time evolution operator $e^{\tau \hat{H}}$ are to be involved. Such calculations are more difficult at lower temperatures. Sota and Tohyama obtained a good accuracy at all temperatures by the RPE, but a further improved treatment of this operator will be discussed in Section IV.5.

\section{Eigenvectors}

The regulated kernel operator (34) serves as an efficient filter to extract an eigenvector component from an arbitrary initial vector. On operating it on a vector $|\xi\rangle$, one obtains

$$
\begin{aligned}
\mid \bar{\xi}> & \equiv\left\langle\delta_{N}(\epsilon-\hat{H})\right\rangle_{\sigma} \mid \xi> \\
& =\sum_{\alpha}\left\langle\delta_{N}\left(\epsilon-\epsilon_{\alpha}\right)\right\rangle_{\sigma}|\alpha><\alpha| \xi>
\end{aligned}
$$

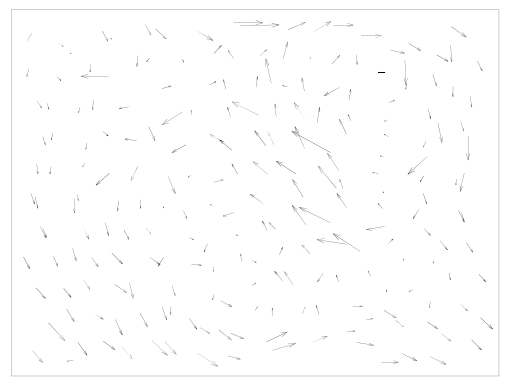

Fig. 7 RPE calculation of an eigenvector of the roton excitation in Ar supercooled liquid. ${ }^{16)}$

Since the weight of each component in the above equation follows the distribution (31), this enhances the amplitudes in the vicinity of $\epsilon$, while eliminating those of the remote values. The renormalization of the vector is taken as granted. Since the width of the weight can be made arbitrarily small and the skirt decays strictly according to the normal distribution, the filtering can be made almost perfect, so that one is able to pick up only the closest component to $\epsilon$. This property of the RPE kernel operator has been exploited by Sota and Itoh ${ }^{16)}$ for studying the vortex-like roton excitations of a glassy material. The result is reproduced in Fig. 7.

For a highly degenerate case, the filtered vector may still have finite amplitudes of the neighbouring components. These residual amplitudes can be eliminated by repeating the above cycle, possibly with a smaller width, by adjusting the parameter $\epsilon$ to $\langle\bar{\xi}|\hat{H}| \bar{\xi}>$ and replacing $| \xi>$ by $\mid \bar{\xi}>$. The above authors attained the accuracy of the six digits, at most in two cycles, for both the eigenvector and the eigenvalue of a $20,000 \times 20,000$ matrix. In the case of a real degeneracy due to symmetry, one may still resolve it completely, by using different initial vectors to obtain different eigenstates, and then adopting Gram-Schmidt orthogonalization to the latter vectors. The same accuracy can not be attained by the KPM Jackson kernel, due to the extending and growing satellite peaks.

\section{Matrix Inversion}

The eigenvector calculation described above suggests that the RPE may be useful for other linear-algebraic problems. Another interesting application is found by setting $z=0$ in Eqs. (7) and (8),

$$
\hat{\Omega}^{-1}=-\sum_{n=0}^{\infty} w_{n}^{-1} \tilde{\varphi}_{n}(0)\left\langle\varphi_{n}(\hat{\Omega})\right\rangle_{\sigma},
$$

where $\tilde{\varphi}_{n}(0)$ refers to the real part of $\tilde{\varphi}_{n}(\epsilon \pm i \delta)$ for $\epsilon=0$. It can be applied, for example, to solving the algebraic equation $\hat{\Omega}|\xi>=| \xi_{0}>$, with the solution being obtainable by the same recursively algorithm as

$$
\left|\xi>=-\sum_{n=0}^{\infty} w_{n}^{-1} \tilde{\varphi}_{n}(0)\left\langle\varphi_{n}(\hat{\Omega})\right\rangle_{\sigma}\right| \xi_{0}>.
$$

This scheme is quite handy and accurate, compared to the existing standard packages, so expected to be useful in many 
areas, not necessarily in physics. In particular, it does not discriminate a dense matrix and was found to work even for a non-hermitian matrix. For example, Yamane and Itoh ${ }^{17)}$ have obtained an accurate solution for a Bethe-Salpeter equation, for which simple iteration failed to converge.

\section{Time Evolution Operator}

Time-dependent approaches in quantum simulations have been studied extensively in recent years, ${ }^{19-23)}$ both in the real and imaginary time domains. Many of them make use of the polynomial expansion to the time evolution operator $e^{-i \hat{H} t}$ or its continuation to the imaginary time. Here we discuss the former operator, because it is visually more illustrative.

In the context of the present paper, it is natural to use the regulated time evolution operator $\left\langle e^{-i \hat{H} t}>_{\sigma}\right.$. This approach has been adopted in References 11-13, but a more accurate and convenient expression is available in the RPE:

$$
\begin{aligned}
e^{-i \hat{H} t} & \simeq e^{\sigma^{2} t^{2} / 2}\left\langle e^{-i \hat{H} t}\right\rangle_{\sigma} \\
& =e^{\sigma^{2} t^{2} / 2} \sum_{n=0}^{N} w_{n}^{-1} \tilde{\varphi}_{n}(t)\left\langle\varphi_{n}(\hat{H})\right\rangle_{\sigma} .
\end{aligned}
$$

The first line of the above expression holds to the same accuracy of Eq. (34) and is proven by using the latter relation, noting that $\left\langle e^{-i \hat{H} t}>_{\sigma}\right.$ is its Fourier transform. Here $\tilde{\varphi}_{n}(t)$ denotes the AF in the time domain, whose explicit expressions are readily obtained for all the polynomials; the case of Legendre is given in Reference 6. It is important to note that the expression (42) is sufficiently accurate for a finite $N$, provided that an optimal $\sigma$ is used, because its accuracy is solely due to Eq. (34) and not to the smallness of $\sigma$. So high precision is expected at relatively low truncation orders.

As a numerical test, we have examined the time evolution of a one-dimensional Gaussian wave packet with a harmonic Hamiltonian

$$
\hat{H}=-\frac{1}{2} \frac{d^{2}}{d x^{2}}+\frac{1}{2}(x-2)^{2}
$$

taking $\psi(x, 0) \propto e^{-(x-a)^{2} / 2 \xi^{2}}$ as the initial state. The reason for studying this model is the availability of an analytic solution. ${ }^{18)}$ We used Hermite polynomial for the expansion (42), for which $\tilde{\varphi}_{n}(t)=(-i t)^{n} \sqrt{\pi} e^{-t^{2} / 4}$. As for constructing the Hamiltinian matrix, we chose the eigenvectors of the undisplaced oscillator as the basis. This representation helps us to reduce the matrix size; we found that 600 eigenstates are sufficient to expand the wave packet. The displaced Hamiltonian (43) is not diagonal in this representation. Although the eigenvectors may be given by Hermite polynomials, our choice of the matrix representation by no means favours the accuracy of the calculation.

Figure 8 is the numerical result for $a=5$ and $\xi=0.5$ of the wave-packet parameters, up to the half-period $(t=\pi)$ of the harmonic motion. The expansion (42) was truncated at $N=650$ and we set $\sigma=C / \sqrt{N}(C=2.94)$. The calculation was found to reproduce the analytic solution very precisely. Although our scheme gives the final state directly in principle, we encounter the numerical underflow for $t$ longer than

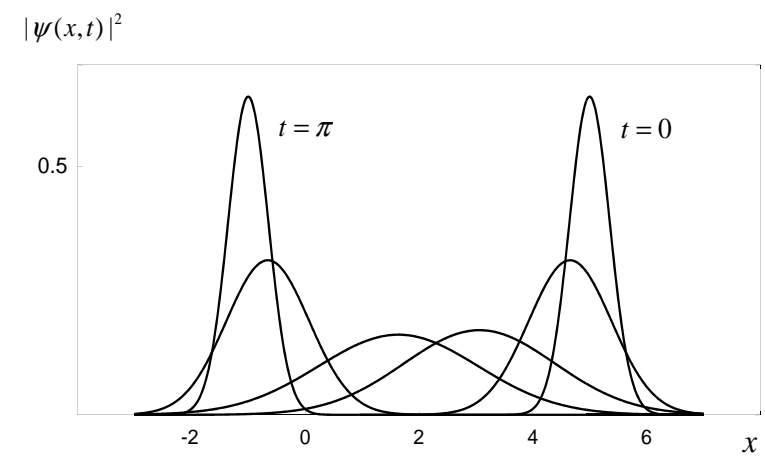

Fig. 8 Probability distribution $|\psi(x, t)|^{2}$ that evoluted from $\psi(x, 0) \propto$ $e^{-(x-a)^{2} / 2 \xi^{2}}(\xi=0.5, a=5.0)$ by the Hamiltonian (43). Calculation has been done by Eq. (42) using Hermite polynomial, with $N=650$ and $\sigma=C / \sqrt{N}(C=2.94)$. From right to left: $t=0,2 \Delta t, 5 \Delta t, 7 \Delta t$, $11 \Delta t$, and $t=\pi$ with $\Delta t=\pi / 13$. The time evolution is strictly periodic, coincident with the analytic solution.

the order of unity. In order to prevent it, we proceeded step by step, by the interval of $\Delta t=2 \pi / 13$, updating the initial state at each step by the final state of the last step. We continued the calculation up to $t=200 \times \pi$, and it produced numerically stable and precisely periodic time evolution, showing no indication of starting to deviate from the analytic solution. Therefore, our methodology appears to assure a very high accuracy with a practical computation time.

The other polynomials can also be used for the time evolution calculation. A more detailed study of this subject will be reported elsewhere. ${ }^{24)}$

\section{Summary}

We have formulated the orthogonal-polynomial expansion in a general framework, particularly of resolvent, elucidating the analytic structure in the frequency plane. Implementation of the RPE technique has been done for all the important polynomials to remove Gibbs oscillation. The advantage in using the RPE is that it achieves both high accuracy and high speed computing with the simplest algorithm, assuring a completely homogeneous resolution. Due to a wide availability of the polynomials, the present generalization has extended the range of the tractable matrices, covering finite, semi-infinite and infinite intervals of the spectrum. It has also found a variety of applications, including correlation functions, eigenvectors, matrix inversion, time evolution and finite temperature calculations. The possible application of the method is expected to be more diverse than shown in this paper.

\section{References}

1) D. W. Bullet, R. Haydoc, V. Heine, M. J. Kelly in H. Ehrenreich, F. Seitz, D. Turnbull (eds.), Solid State Physics, Vol. 35, Academic Press, New York (1980).

2) A. F. Voter, J. D. Kress, R. N. Silver, Phys. Rev., B53, 12733(1996).

3) R. Chen, H. Guo, Comp. Phys. Commun., B119, 19 (1999).

4) A. Weisse, G. Wellein, A. Alvermann, H. Fehske, Rev. Mod. Phys., 78, 275 (2006). and references therein. 
5) R. N. Silver, H. Röder, Phys. Rev., E56 4822 (1997).

6) S. Sota, M. Itoh, J. Phys. Soc. Jpn., 76[5], 054004 (2007).

7) S. Sota, M. Itoh, J. Korean Phys. Soc., 54[915], 386-392 (2009).

8) M. Amini, S. A. Jafari, F. Shahbazi, Europhys. Lett., 87, 37002 (2009).

9) J. Schleede, G. Schubert, H. Fehske, Europhys. Lett., 90, 17002 (2010).

10) T. Iitaka, T. Ebisuzaki, Phys. Rev. Lett., 90, 047203 (2003).

11) S. Sota, T. Tohyama, Phys. Rev., B78, 113101 (2008).

12) S. Sota, T. Tohyama, J. Phys. Conf. Ser., 200, 012191 (2010).

13) T. Sugimoto, S. Sota, T. Tohyama, Phys. Rev., B82, 035437 (2010).

14) P. M. Morse, H. Feshbach, "Method of Theoretical Physics", McGraw-Hill, CH5 (1953).

15) M. Itoh, Phys. Rev., B15 4241 (1992).

16) S. Sota, M. Itoh, J. Korean Phys. Soc., 54[915], 393-399 (2008).

17) Y. Yamane, M. Itoh, in preparation.

18) R. P. Feynman, A. R. Hibbs, in Quantum Mechanics and Path Integrals, McGrow-Hill (1965).

19) S. Roche, D. Mayou, Phys. Rev. Lett., B79, 2518 (1997).

20) M. L. Williams, J. Maris, Phys. Rev., B31, 4508 (1985).

21) K. Yakubo, T. Nakayama, J. Maris, J. Phys. Soc. Jpn., B60, 3249 (1991).

22) T. Iitaka, "Computing the real-time Green's Function of large Hamiltonian matrices," High performance Computing in RIKEN 1995, ISSN-1342-3428, 241 (1996).

23) T. Iitaka, S. Nomura, H. Hirayama, X. Zhao, Y. Aoyagi, T. Sugano, Phys. Rev., B56, 1222 (1997).

24) Matsumoto et al., in preparation.

\section{Appendix}

\section{A Determination of Adjoint Functions and their An- alytic Structure}

In this appendix we complement the argument in Section II.2, by giving a thorough discussion of the ADE solutions and their analytical continuations $\tilde{\varphi}_{n}(\epsilon \pm i \delta)$. This elucidate the mathematical structure of the polynomial expansion in the complex frequency plane. It also gives the correct analytic expressions for the initial terms tabulated in Appendix B.

\section{Adjoint Differential Equations and Bilinear Concomi- tant}

The argument in Section II.2 relies on a special property of the Hilbert transformation (17). By this transformation, a canonical second order differential equation $\mathcal{L}_{z} \psi(z)=0$ is written by partial integrations as

$$
\begin{aligned}
\int_{a}^{b} d x(z & -x)^{-1} \mathcal{L}_{x} v(x) \\
& +\left.P(z, x, v)\right|_{x=b}-\left.P(z, x, v)\right|_{x=a}=0,
\end{aligned}
$$

where the functional $P(z, x, v)$ represents the marginal contributions, and $\mathcal{L}_{x}$ denotes the same operator as $\mathcal{L}_{z}$, with the variable being replaced.
The functional $P$ is called the bilinear concomitant. ${ }^{14)}$ If it vanishes at the boundary, then the two functions $\psi(z)$ and $v(x)$ satisfy the same differential equation. Indeed one can prove $\left.P(z, x, v)\right|_{x=a, b}=0$ when $v(x)=w(x) \varphi_{n}(x)$, with $\varphi_{n}(x)$ being any of the orthogonal polynomials dealt with in this paper and $\mathcal{L}_{z}$ the differential operator such that $\mathcal{L}_{x}\left\{w(x) \varphi_{n}(x)\right\}=0$. We give below the proof in the case of Jacobi polynomial. This polynomial satisfies the differential equation

$$
\begin{aligned}
& \left(x^{2}-1\right) \frac{d^{2}}{d x^{2}} P_{n}^{\alpha \beta}(x) \\
& +[(\alpha+\beta+2) x+(\alpha-\beta)] \frac{d}{d x} P_{n}^{\alpha \beta}(x) \\
& \quad-n(n+\alpha+\beta+1) P_{n}^{\alpha \beta}(x)=0 .
\end{aligned}
$$

Knowing that $w(x)=(1-x)^{\alpha}(1+x)^{\beta}$, the operator $\mathcal{L}_{z}$ is readily identified and the ADE (18) results. We then obtain the bilinear concomitant explicitly,

$$
\begin{aligned}
P(z, x, v)= & (1-x)^{1+\alpha}(1+x)^{1+\beta} \\
& \times\left\{\frac{P_{n}^{\alpha \beta}(x)}{(z-x)^{2}}+\frac{P_{n}^{\prime \alpha \beta}(x)}{(z-x)}\right\},
\end{aligned}
$$

by performing the partial integrations. Noting that $\alpha, \beta>-1$, it clearly vanishes at $x= \pm 1$.

The above proof covers the cases of Legendre, Tchebyshev and Gegenbauer polynomials. The proof goes almost the same way for Hermite and Laguerre polynomials.

\section{Analytical Continuation for Jacobi Polynomial}

Knowing the differential equation for the polynomial, we can readily derive the ADE. In the case of Jacobi, Eq. (18) results. Taking again this case as a representative, we describe in detail how the analytic continuation $\tilde{\varphi}_{n}(\epsilon \pm i \delta)$ is obtained, assuming that the two indices $\alpha, \beta$ have non-integral values.

Equation (18) is canonical, with the three singularities at $z= \pm 1$ and $z=\infty$. The Papperitz indices are readily identified. Using the Riemann symbol, the general solution is denoted as

$$
\tilde{P}_{n}^{\alpha \beta}(z)=P\left\{\begin{array}{ccc}
-1 & +1 & \infty \\
0 & 0 & n+1 \\
\beta & \alpha & -(n+\alpha+\beta)
\end{array}\right\} .
$$

Since we first wish to obtain the expression about the infinity, we transform it into the reduced form, i.e. shift the singularities to $z=0,1$ and $\infty$. This is done by changing the variable $z$ either to $(1+z) / 2$ or $(1-z) / 2$, and the Papperitz indices remain unchanged by these transformations.

Using the former variable, we can immediately see that the solution compatible with the asymptotic behaviour (14) is given by

$$
\begin{aligned}
\tilde{P}_{n}^{\alpha \beta}(z) & \propto\left(\frac{1}{1+z}\right)^{n+1} \\
& \times F\left(n+1, n+\beta+1|2 n+\alpha+\beta+2| \frac{2}{1+z}\right) \\
& \sim 1 / z^{n+1},
\end{aligned}
$$


where $F$ is the hypergeometric function. The constant of the proportionality in the first line must be $K_{n}^{(n)}$ in accordance with Eq. (14). It is calculated from Eq. (13) as

$$
K_{n}^{(n)}=\frac{2^{n+\alpha+\beta+1} \Gamma(\alpha+n+1) \Gamma(\beta+n+1)}{\Gamma(\alpha+\beta+2 n+2)} .
$$

Representing the hypergeometric function as a series expansion, the solution (48) is convergent only outside the left circle of radius two, shown in Fig. 1, centered on $z=-1$. Therefore it must be analytically continued into the circle (region I). This is done by using the joining equation connecting the two hypergeometric functions around $z=\infty$ and $z=0$, with the variable $z$ being replaced by $(1+z) / 2$. In order to write it in a form involving the trivial solution $w(z) \varphi_{n}(z)$, we further make use of another joining equation referring to the two singular points on the real axis. The result is

$$
\begin{aligned}
\tilde{P}_{n}^{\alpha \beta}(z)= & \frac{\pi}{\sin \pi \beta} \cdot(1-z)^{\alpha}(-1-z)^{\beta} P_{n}^{\alpha \beta}(z) \\
+ & \frac{(-1)^{n+1} 2^{\alpha+\beta} \Gamma(n+\alpha+1) \Gamma(\beta)}{\Gamma(n+\alpha+\beta+1)} \\
& \times F\left(n+1,-\alpha-\beta-n|1-\beta| \frac{1+z}{2}\right),
\end{aligned}
$$

where the first term comes from

$P_{n}^{\alpha \beta}(z)=\frac{\Gamma(n+1+\alpha)}{n ! \Gamma(1+\alpha)} F\left(-n, n+\alpha+\beta+1|\alpha+1| \frac{1-z}{2}\right)$,

using the relation $\Gamma(-t) \Gamma(t+1)=-\pi / \sin \pi t$.

In exactly the same manner, an alternative expression is obtained by choosing $(1-z) / 2$ as the variable:

$$
\begin{aligned}
\tilde{P}_{n}^{\alpha \beta}(z)= & -\frac{\pi}{\sin \pi \alpha} \cdot(-1+z)^{\alpha}(1+z)^{\beta} P_{n}^{\alpha \beta}(z) \\
+ & \frac{2^{\alpha+\beta} \Gamma(n+\beta+1) \Gamma(\alpha)}{\Gamma(n+\alpha+\beta+1)} \\
& \times F\left(n+1,-\alpha-\beta-n|1-\alpha| \frac{1-z}{2}\right) .
\end{aligned}
$$

This expression is valid in region II in Fig. 1. By using Kummer's relation, the two expressions are shown to be identical in the common convergence region (the hatched area in Fig. 1). The analytic continuation is then carried on, down to the real axis, to yield the two alternative expressions as shown in Eqs. (19). They are identical as they should be, and this gives the complete solution to the problem.

There is a subtle point in the above derivation about the branch cut. The function $F(a, b|c| 1 / z)$ has a cut along the real axis from $z=0$ to $z=1$, so the joining equations must be used for Eq. (48) taking this constraint into account. This specifies the Riemann sheet to $-\pi<\arg (-z) \leq+\pi$, with a cut between $z=-1$ and $z=+1$ as is shown in Fig. 1, in either case of Eqs. (50) and (52). It prevents these two functions to be multi-valued for the non-integral indices.

So far we have assumed the indices $\alpha$ and $\beta$ to be both non-integers. To be precise, Eq. (50) is valid only for a nonintegral value of $\beta$, whereas Eq. (52) for a non-integral $\alpha$. Otherwise the respective terms involve contributions that blow up, so do the corresponding terms in Eqs. (19). This also applies to the Legendre polynomial $(\alpha=\beta=0)$, and we discuss this case separately below. In fact this divergence is a spurious one; they are shown to cancel each other. Anyhow it does not bother us in the recursive algorithm described in Section II.3, which covers all the cases on equal footings.

\section{Analytical Continuation for Other Polynomials}

\section{(1) Legendre Polynomial $P_{n}(x)$}

Legendre polynomial belongs to the family of Jacobi, but we can apply different joining equations in this particular case to obtain

$$
\begin{aligned}
\tilde{P}_{n}(z) & =\frac{(n !)^{2}}{(2 n+1) !} \frac{2^{n+1}}{z^{n+1}} F\left(\frac{n+1}{2}, \frac{n+2}{2}, n+\frac{3}{2} \mid \frac{1}{z^{2}}\right) \\
& \equiv 2 \mathcal{Q}_{n}(z),
\end{aligned}
$$

where the function $\mathcal{Q}_{n}(z)$ is called the Legendre function of the second kind. The terminology of the "second kind" often refers only to the real part of $\mathcal{Q}_{n}(\epsilon \pm i \delta)$. Denoting it by $Q_{n}(\epsilon)$, we have a divergence-free expression

$$
\tilde{P}_{n}(\epsilon \pm i \delta)=2 Q_{n}(\epsilon) \mp i \pi P_{n}(\epsilon)
$$

which coincides with the familiar definition

$$
Q_{n}(\epsilon)=\frac{1}{2} \mathcal{P} \int_{-1}^{1} \frac{P_{n}(x)}{\epsilon-x} d x .
$$

(2) Tchebyshev and Gegenbauer Polynomials $T_{n}(x), C_{n}^{\gamma}(x)$

Jacobi polynomials with symmetric indices $(\alpha=\beta)$ are usually distinguished by the name of Gegenbauer polynomial, adopting a different normalization

$$
C_{n}^{\gamma}(x)=\frac{\Gamma(n+2 \gamma) \Gamma(\gamma+1 / 2)}{\Gamma(2 \gamma) \Gamma(n+\gamma+1 / 2)} P_{n}^{\gamma-1 / 2 \gamma-1 / 2}(x) .
$$

This leads to a simpler recurrence relation, but the results are basically the same as already given for Jacobi.

Exception is the case of $\gamma=0$, for which $C_{n}^{\gamma}(x)$ is illdefined and an yet different normalization is to be adopted:

$$
T_{n}(x)=\frac{\left(2^{n} n !\right)^{2}}{(2 n) !} P_{n}^{-1 / 2-1 / 2}(x) .
$$

This is Tchebyshev polynomial, and the corresponding AF is

$$
\tilde{T}_{n}(\epsilon \pm i \delta)=-\pi\left(1-\epsilon^{2}\right)^{-1 / 2}\left[V_{n}(\epsilon) \pm i T_{n}(\epsilon)\right]
$$

where

$$
V_{n}(\epsilon)=\frac{2^{n-1} n !}{(2 n-1) ! !}\left(1-x^{2}\right)^{1 / 2} P_{n-1}^{1 / 21 / 2}(\epsilon)
$$


is the second kind solution of the Tchebyshev equation $\left(V_{0}(\epsilon)=0\right.$ by definition). A remarkable feature of this polynomial is the simplification of Eq. (58),

$$
\begin{aligned}
\tilde{T}_{n}(\epsilon \pm i \delta) & =\mp i \pi\left(1-\epsilon^{2}\right)^{-1 / 2}\left[\epsilon \mp i\left(1-\epsilon^{2}\right)^{1 / 2}\right]^{n} \\
& =\frac{\mp i \pi e^{\mp i n \theta}}{\sin \theta} \quad(\epsilon=\cos \theta)
\end{aligned}
$$

a property which has been extensively exploited in the KPM. ${ }^{3)}$

\section{(3) Hermite Polynomial $H_{n}(x)$}

The definition interval of this polynomial is $(-\infty, \infty)$, and two different conventions are commonly used for the weight function; $w(x)=e^{-x^{2}}$ or $=e^{-x^{2} / 2}$. We adopt the former. The corresponding ADE is

$$
\tilde{H}_{n}^{\prime \prime}(z)+2 z \tilde{H}_{n}^{\prime}(z)+2(n+1) \tilde{H}_{n}(z)=0 .
$$

This is a differential equation without singularities. However it can be transformed to the form of a confluent hypergeometric equation by setting $\zeta=-z^{2}$,

$$
\left\{\zeta \frac{d^{2}}{d \zeta^{2}}+(1 / 2-\zeta) \frac{d}{d \zeta}-\frac{n+1}{2}\right\} \tilde{H}_{n}=0
$$

The two independent solutions of the above equation are $F(n / 2+1 / 2,1 / 2 \mid \zeta)$ and $z F(n / 2+1,3 / 2 \mid \zeta)$, where $F(a, c \mid \zeta)$ denotes the confluent hypergeometric function. Here again we must take into account the asymptotic condition (14) for taking their linear combination. It is, however, less trivial in the present case, because these two solutions are known to show "Stokes' phenomenon". That is, they have different asymptotic behaviours, depending on the directions pointing to the infinity. ${ }^{14)}$ The two linear combinations which suppress the Stokes phenomenon are the confluent hypergeometric functions of the third kind, ${ }^{14)}$

$$
\begin{aligned}
& U_{1}\left(\frac{n+1}{2}, \frac{1}{2} \mid \zeta\right) \sim \zeta^{-n / 2} e^{-\zeta} \\
& U_{2}\left(\frac{n+1}{2}, \frac{1}{2} \mid \zeta\right) \sim \zeta^{-(n+1) / 2} e^{i \frac{\pi}{2}(n+1)} .
\end{aligned}
$$

The second function meets the asymptotic condition (14). In determining the proportionality constant, the phase of the half-integral power must be chosen carefully, considering the branch cut between $\zeta=0$ and $\zeta=-\infty$. We obtain

$$
\tilde{H}_{n}(z)=(-1)^{n+1} n ! \pi^{1 / 2} U_{2}\left(\frac{n+1}{2}, \frac{1}{2} \mid \zeta\right) .
$$

This expression is rewritten by a linear combination of the two confluent hypergeometric functions. In doing so, it is to be recalled that the variable $\zeta$ crosses the cut of $U_{2}$ when $z$ crosses the real axis. Therefore one must use different phase factors for the respective terms on the second Riemann sheet. Explicit expressions for $\tilde{H}_{n}(\epsilon \pm i \delta)$ are then obtained separately for even and odd degrees:

$$
\begin{aligned}
& \tilde{H}_{2 n}(\epsilon \pm i \delta)=\mp i \pi e^{-\epsilon^{2}} H_{2 n}(\epsilon) \\
& +(-1)^{n} \pi^{1 / 2} 2^{2 n+1} n ! \epsilon F\left(n+1,3 / 2 \mid-\epsilon^{2}\right) \\
& \tilde{H}_{2 n+1}(\epsilon \pm i \delta)=\mp i \pi e^{-\epsilon^{2}} H_{2 n+1}(\epsilon) \\
& \quad-(-1)^{n} \pi^{1 / 2} 2^{2 n+1} n ! \epsilon F\left(n+1,1 / 2 \mid-\epsilon^{2}\right) .
\end{aligned}
$$

Apart from the numerical factors, the real parts of Eqs. (65) are identical to the Hermite functions of the second kind times the weight function.

\section{(4) Laguerre Polynomial $L_{n}^{\alpha}(x)$}

This polynomial is defined in the interval of $0<x<\infty$ with $w(x)=x^{\alpha} e^{-x}, \alpha>-1$. The $\mathrm{ADE}$ is given by

$z \frac{d^{2}}{d z^{2}} \tilde{L}_{n}^{\alpha}(z)+[z+(1-\alpha)] \frac{d}{d z} \tilde{L}_{n}^{\alpha}(z)+(n+1) \tilde{L}_{n}^{\alpha}(z)=0$

and takes the form of confluent hypergeometric equation by choosing $-z$ as a variable. Taking again the function $U_{2}$ for the solution and determining the constant by Eq. (14), we have

$$
\tilde{L}_{n}^{\alpha}(z)=(-1)^{n} \Gamma(n+\alpha+1) U_{2}(n+1,1-\alpha \mid-z) .
$$

The expression is valid for all values of $\alpha>-1$. When $\alpha$ is not an integer, we obtain

$$
\begin{aligned}
& \tilde{L}_{n}^{\alpha}(\epsilon \pm i \delta)=(\pi \cot \pi \alpha \mp i \pi) \epsilon^{\alpha} e^{-\epsilon} L_{n}^{\alpha}(\epsilon) \\
& -\Gamma(\alpha) F(n+1,1-\alpha \mid-\epsilon) \text {. }
\end{aligned}
$$

When $\alpha$ is an integer, we have the same divergence as we have met in the case of Jacobi polynomial, which is again handled properly in the recursive calculation.

\section{B Table for the Recursive Calculation}

For practical application of the RPE for the resolvent expansion, we only needs $\tilde{\varphi}_{0}(\epsilon \pm i \delta)$ and the coefficients for the simultaneous recurrence formulae, particularly $A_{n}^{\prime}, B_{n}^{\prime}$ and $C_{n}^{\prime}$ in Eq. (29). We list them below for all the polynomials studied in this paper. Other parameters are found in standard textbooks. In this table it is understood that $C_{0}^{\prime}=0$ for all cases, and the index $\alpha$ or $\beta$ is assumed not to be an integer. The results for the integral indices are given by Eqs. (23) and (24). For using Hermite and Laguerre polynomials, it is recommended to normalize them to unity prior to regulation, in order to reduce numerical overflow.

$$
\begin{aligned}
& \text { Legendre } P_{n}(x): \quad w(x)=1 \\
& \qquad \begin{array}{c}
A_{n}^{\prime}=2 n+1, \quad B_{n}^{\prime}=0, \quad C_{n}^{\prime}=1 \\
\tilde{P}_{0}(\epsilon \pm i \delta)=\ln \left|\frac{\epsilon+1}{\epsilon-1}\right| \quad \mp i \pi
\end{array}
\end{aligned}
$$


Tchebyshev $T_{n}(x): \quad w(x)=\left(1-x^{2}\right)^{-1 / 2}$

$$
\begin{aligned}
& A_{n}^{\prime}=2(n+1), \quad B_{n}^{\prime}=0, \quad C_{n}^{\prime}=\frac{n+1}{n-1} \quad\left(A_{0}^{\prime}=1\right) \\
& \tilde{T}_{0}(\epsilon \pm i \delta)=\mp i \pi w(\epsilon)
\end{aligned}
$$

Jacobi $P_{n}^{\alpha \beta}(x): \quad w(x)=(1-x)^{\alpha}(1+x)^{\beta}$

$$
\begin{aligned}
A_{n}^{\prime}= & \frac{(2 n+\alpha+\beta+1)(2 n+\alpha+\beta+2)}{2(n+\alpha+\beta+1)} \\
B_{n}^{\prime}= & -\frac{(\alpha-\beta)(2 n+\alpha+\beta+1)}{(n+\alpha+\beta+1)(2 n+\alpha+\beta)} \\
C_{n}^{\prime}= & \frac{(n+\alpha)(n+\beta)(2 n+\alpha+\beta+2)}{(n+\alpha+\beta)(n+\alpha+\beta+1)(2 n+\alpha+\beta)} \\
\left.\tilde{P}_{0}^{\alpha \beta}(\epsilon) \pm i \delta\right) & =w(\epsilon)(-\pi \cot \pi \alpha \mp i \pi) \\
& +\frac{2^{\alpha+\beta} \Gamma(\alpha) \Gamma(\beta+1)}{\Gamma(\alpha+\beta+1)} F\left(1,-\alpha-\beta|1-\alpha| \frac{1-\epsilon}{2}\right) \\
= & w(\epsilon)(\pi \cot \beta \mp i \pi) \\
& -\frac{2^{\alpha+\beta} \Gamma(\beta) \Gamma(\alpha+1)}{\Gamma(\alpha+\beta+1)} F\left(1,-\alpha-\beta|1-\beta| \frac{1+\epsilon}{2}\right)
\end{aligned}
$$

Laguerre $L_{n}^{\alpha}(x): \quad w(x)=x^{\alpha} e^{-x}$

$$
\begin{aligned}
& A_{n}^{\prime}=-1, \quad B_{n}^{\prime}=1, \quad C_{n}^{\prime}=0 \\
& \tilde{L}_{0}^{\alpha}(\epsilon \pm i \delta)=w(\epsilon)\left(\begin{array}{rl}
\pi \cot \pi \alpha \mp i \pi) \\
-\Gamma(\alpha) F(1,1-\alpha \mid-\epsilon)
\end{array}\right.
\end{aligned}
$$

Hermite $H_{n}(x): \quad w(x)=e^{-x^{2}}$

$$
\begin{aligned}
& A_{n}^{\prime}=2(n+1), \quad B_{n}^{\prime}=0, \quad C_{n}^{\prime}=0 \\
& \tilde{H}_{0}(\epsilon \pm i \delta)=2 \sqrt{\pi} \epsilon F\left(1,3 / 2 \mid-\epsilon^{2}\right) \mp i \pi w(\epsilon)
\end{aligned}
$$

